\title{
Amine facilitates the synthesis of silica-supported ultrasmall bimetallic nanoparticles
}

\author{
Nanfeng Zheng ${ }^{*}$ and Pengxin Liu
}

Supported catalysts based on metal nanoparticles are a class of widely used heterogeneous catalysts in industry. The catalytic performances of supported metal catalysts are highly determined by many parameters of metal nanoparticles such as their particle size, composition, surface structure and also interfacial interaction with supports [1]. Supported metal catalysts are commonly prepared by depositing metal precursors on high-surface area supports through various methods such as impregnation (IMP), co-precipitation (CP), and depositionprecipitation (DP), followed by thermal or chemical reduction of the metal precursors deposited thereon to form supported metal nanoparticles.

The difficulty for conventional methods to prepare high-quality supported metal nanoparticles with uniform particle size and composition mainly lies in the following two aspects: 1) The interaction between metal precursors and supports is not strong enough to prevent their sintering during the post thermal or chemical reduction processes; 2) The deposition of metal precursors is not homogenous so that the phase separation often occurs after the reducing treatment. By addressing these two issues, the DP method has been outstanding among other conventional methods in preparing supported small metal nanoparticles [2]. However, the DP method has met great difficulties in preparing high-quality metal nanoparticles catalysts on many acidic supports (such as silica, $\mathrm{WO}_{x}$, zeolites) due to their relatively low point of zero charge (PZC) [3]. During the past decade, there has thus been an emergence of research efforts directed toward the use of pre-made uniform nanoparticles as precursors to prepare well-controlled metal nanocatalysts for investigating complicated interfacial catalytic mechanism [4-6]. In these colloidal methods, the influence of organic capping agents is often concerned.

As compared to supported monometallic nanoparticles, the preparation of high-quality supported alloyed nanoparticles is even more challenging. Alloying has been well-documented as an effective strategy to tailor the catalytic performance of supported metal catalysts. On one hand, alloying provides an avenue to reduce the usage of expensive scare metal without compromising the overall catalytic activity. On the other hand, the electronic density and/or dispersion of catalytically active metal can be manipulated by the introduction of another metal species, thus tailoring the reaction pathway and often optimizing the overall catalytic properties. In order to obtain high-quality supported alloyed catalysts with uniform ultrasmall size and uniform composition, it is essential to have the metal precursors uniformly deposited onto the surface of supports before being converted into alloyed nanoparticles.

Recently, Regalbuto and colleagues have developed a general and simple method to prepare ultrasmall alloyed metal nanoparticles based on both noble (i.e., Pt, Pd) and base metals (i.e., Ni, Co, Cu) [7]. The homogeneously alloyed metal nanoparticles have a narrow size distribution around $1 \mathrm{~nm}$. In the developed method, positively charged metal-amine complex precursors were deposited onto negatively charged surface of silica support through strong electrostatic adsorption (SEA) [8,9], and then reduced by $\mathrm{H}_{2}$ at an elevated temperature to give supported uniform ultrasmall alloyed nanoparticles (Fig. 1a). The key of this work is to use positively charged metal-amine complexes as metal precursors. Since the surface point of zero charge of silica (Aerosil 300 silica) is around 3.6, the surface charge of silica in water is negative in a wide range of $\mathrm{pH}$ value (4-13) before silica is dissolved. The strong electrostatic interaction can thus promote the homogenous deposition, making the method outstanding conventional preparation methods for the production of ultrasmall alloyed nanoparticles with different combina-

Collaborative Innovation Center of Chemistry for Energy Materials, State Key Laboratory for Physical Chemistry of Solid Surfaces, and Department of Chemistry, College of Chemistry and Chemical Engineering, Xiamen University, Xiamen 361005, China

"Email: nfzheng@xmu.edu.cn 
a
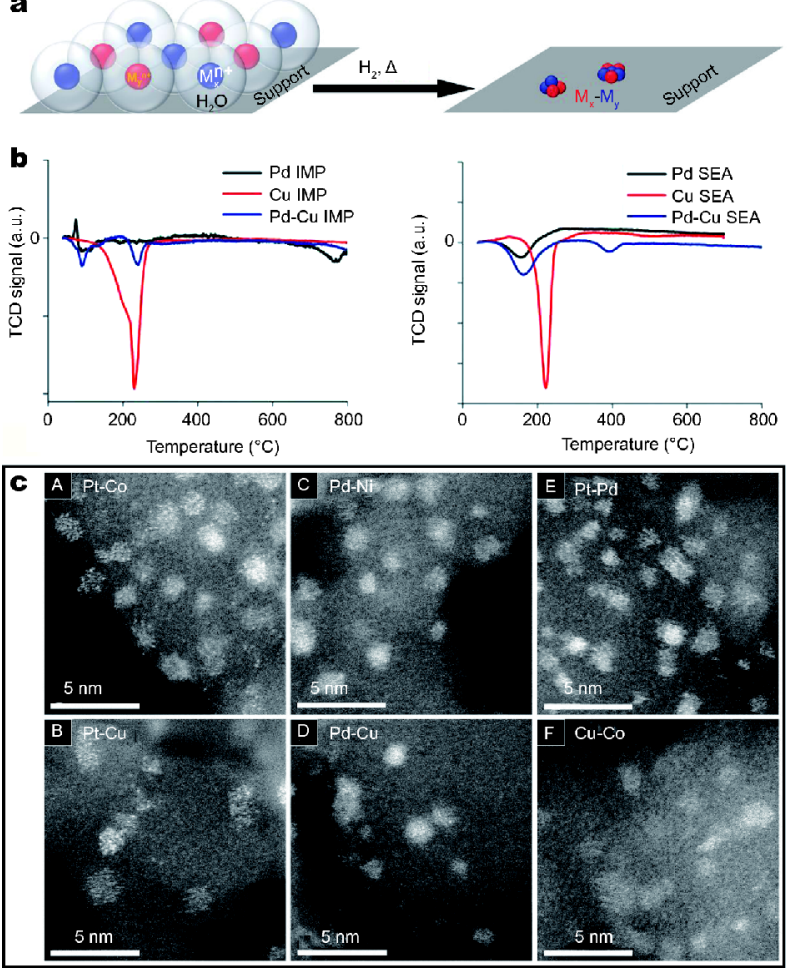

Figure 1 The synthesis of $\mathrm{SiO}_{2}$-supported ultrasmall alloyed bimetallic nanoparticles based on strong electrostatic adsorption: (a) The scheme showing the preparation method, (b) the comparison TPR profiles (downward peaks indicate $\mathrm{H}_{2}$ consumption) of the samples with $\mathrm{Pd}$ and $\mathrm{Cu}$ precursors deposited on $\mathrm{SiO}_{2}$ by the IMP and SEA methods, (c) the atomically resolved Z-contrast images of as-prepared ultrasmall alloyed nanoparticles. Scale bars, $5 \mathrm{~nm}$. Reproduced with permission from [7], Copyright 2017, American Association for the Advancement of Science.

tions of metals.

The concept of simultaneous deposition of two different metal-amine complex precursors on silica was first demonstrated in the work by systematically investigating the adsorption behaviors of ten different metal pairs (i.e., Pt-Pd, Pd-Cu, Ni-Co, Pt-Ni, Pt-Cu, Pt-Co, Pd-Ni, Ni-Cu, $\mathrm{Pd}-\mathrm{Co}$ and $\mathrm{Co}-\mathrm{Cu}$ ) under different $\mathrm{pH}$ values. The uptake surveys revealed the metal- and $\mathrm{pH}$-dependent adsorption behaviors. But the maximum adsorption of all the ten bimetallic combination was about 1.0 to $1.3 \mu \mathrm{mol} \mathrm{m}^{-2}$, eliminating the difference among metals for the creation of a large variety of bimetallic combinations.

After the studies on the co-adsorption of metal-amine precursor pairs, temperature-programmed reduction (TPR) was used to characterize the intimacy of metal alloying in the nascent nanoparticles. The reduction properties of monometallic and bimetallic precursors deposited on silica by IMP and SEA methods were compared (Fig. 1b). The reduction peak(s) of the monometallic samples prepared by SEA were generally shifted to higher temperature as compared to those prepared by IMP. The authors attributed the observed temperature increase of the reduction peaks to a much stronger interaction between the metal precursors and support in SEA than that in IMP. For example, while Pd in the Pd IMP sample was reduced at $\sim 100^{\circ} \mathrm{C}$, the Pd SEA sample required $\sim 150^{\circ} \mathrm{C}$ for the reduction. Moreover, when alloying with noble metal such as Pd, significant shifts of the reduction peak(s) of base-metal (e.g., $\mathrm{Cu}, \mathrm{Co}$ ) precursors towards much lower temperature were observed in the co-SEA samples. For instance, the combination of $\mathrm{Pd}$ and $\mathrm{Cu}$ shifted the reduction peak of $\mathrm{Cu}$ from $500^{\circ} \mathrm{C}$ to $400^{\circ} \mathrm{C}$. In comparison, there were obvious shifts for the reduction peaks of bimetallic samples prepared as compared to those peaks from the monometallic precursors. The shift of the reduction peaks were explained by hydrogen spillover from the noble metal centers to their nearby unreduced base metal species, facilitating the formation of uniform ultrasmall alloyed nanoparticles.

Due to the lack of strong interactions between the metal precursors and the support, metal nanoparticles in the catalysts prepared by the IMP method are typically large and non-uniform in size. In contrast, the electron microscopy Z-contract imaging studies revealed that the alloyed nanoparticles in the bimetallic catalysts prepared by the SEA method had a rather narrow particle size distribution in the range of $\sim 1.0$ to $1.3 \mathrm{~nm}$ (Fig. 1c), nicely explaining the absence of X-ray diffraction (XRD) peaks for the bimetallic catalysts made by the SEA method. More impressively, energy-dispersive X-ray spectroscopy (EDXS) mapping and atomically resolved Z-contrast images demonstrated the homogenous distribution of different metals within the obtained well-alloyed nanoparticles with such an ultrasmall diameter.

At the end of the paper, the authors pointed out that this method had several drawbacks. For instance, silica may dissolve at high $\mathrm{pH}$ values. The loading content of the charged precursors was calculated to be about $1 \mu \mathrm{mol} \mathrm{m}{ }^{-2}$ corresponding to a steric maximum of closepacked complexes with one to two hydration sheathes. Hence, the metal loading is restricted by the surface area of the support. What one should keep in mind is that the metal loading is already in the range of $\sim 1-4 \mathrm{wt} \%$ although the surface area on the silica support used in the study is only $200 \mathrm{~m}^{2} \mathrm{~g}^{-1}$. Therefore, when supports with higher surface areas are used, one should not have problem to increase the metal loading. More importantly, 
together with the easy removal of amine and thus no need to worry about its poisoning effect on catalysis, the positive charge and good stability of metal-amine complex precursors makes the reported SEA and co-SEA methods highly promising for creating ultrafine nanoparticles on a variety of supports, which deserves more systematic studies. Further studies should also be carried out to evaluate the unique feature of such ultrasmall metal nanoparticles in catalysis and their stability under realistic catalysis conditions.

Received 9 January 2018; accepted 12 January 2018;

published online 25 January 2018

1 Corma A, Garcia H. Supported gold nanoparticles as catalysts for organic reactions. Chem Soc Rev, 2008, 37: 2096

2 Haruta M. Gold as a novel catalyst in the 21st century: Preparation, working mechanism and applications. Gold Bull, 2004, 37: 27-36
3 Parks GA. The isoelectric points of solid oxides, solid hydroxides, and aqueous hydroxo complex systems. Chem Rev, 1965, 65: 177198

4 Wu Y, Wang D, Li Y. Nanocrystals from solutions: catalysts. Chem Soc Rev, 2014, 43: 2112-2124

5 Li YD. Nanoparticle chemistry upgrades catalytic interfaces in noble metal catalysts. Sci China Chem, 2014, 57: 924-925

6 Chen G, Zhao Y, Fu G, et al. Interfacial effects in iron-nickel hydroxide-platinum nanoparticles enhance catalytic oxidation. Science, 2014, 344: 495-499

7 Wong A, Liu Q, Griffin S, et al. Synthesis of ultrasmall, homogeneously alloyed, bimetallic nanoparticles on silica supports. Science, 2017, 358: 1427-1430

8 Lambert S, Job N, Dsouza L, et al. Synthesis of very highly dispersed platinum catalysts supported on carbon xerogels by the strong electrostatic adsorption method. J Catal, 2009, 261: 23-33

9 Zečević J, Vanbutsele G, de Jong KP, et al. Nanoscale intimacy in bifunctional catalysts for selective conversion of hydrocarbons. Nature, 2015, 528: 245-248 